\title{
Nontrivial solutions for a fractional boundary value problem
}

Keyu Zhang ${ }^{1,2^{*}}$ and Jiafa $X u^{2}$

\author{
"Correspondence: \\ sduzky@163.com; \\ keyu_292@163.com \\ 1 Department of mathematics, Qilu \\ Normal University, Jinan, Shandong \\ 250013, China \\ ${ }^{2}$ School of Mathematics, Shandong \\ University, Jinan, Shandong 250100 \\ China
}

\author{
Abstract \\ In this work, we discuss the existence of nontrivial solutions for the fractional \\ boundary value problem

$$
\left\{\begin{array}{l}
D_{0+}^{\alpha} u=-f(t, u), \quad t \in[0,1], \\
u(0)=u^{\prime}(0)=u^{\prime}(1)=0 .
\end{array}\right.
$$

Here $\alpha \in(2,3]$ is a real number, $D_{0+}^{\alpha}$ is the standard Riemann-Liouville fractional derivative of order $\alpha$. By virtue of some inequalities associated with Green's function, without the assumption of the nonnegativity of $f$, we utilize topological degree theory to establish our main results.

MSC: 26A33; 34B15; 34B18; 34B27

Keywords: fractional boundary value problem; nontrivial solution; topological degree; Riemann-Liouville derivative

\section{Introduction}

In this paper, we investigate nontrivial solutions for the boundary value problem of fractional order involving Riemann-Liouville's derivative

$$
\left\{\begin{array}{l}
D_{0+}^{\alpha} u=-f(t, u), \quad t \in[0,1] \\
u(0)=u^{\prime}(0)=u^{\prime}(1)=0
\end{array}\right.
$$

where $\alpha \in(2,3], f:[0,1] \times \mathbb{R} \rightarrow \mathbb{R}(\mathbb{R}:=(-\infty,+\infty))$ is continuous.

In view of a fractional differential equation's modeling capabilities in engineering, science, economy and other fields, the last few decades have resulted in a rapid development of the theory of fractional differential equation; see the books [1-3]. This may explain the reason why the last few decades have witnessed an overgrowing interest in the research of such problems, with many papers in this direction published. We refer the interested reader to [4-21] and the references therein.

In [4], Bai and Lü studied the existence and multiplicity of positive solutions for the nonlinear fractional differential equation

$$
\left\{\begin{array}{l}
D_{0+}^{\alpha} u(t)+f(t, u(t))=0, \quad 0<t<1, \\
u(0)=u(1)=0,
\end{array}\right.
$$

๑ 2013 Zhang and Xu; licensee Springer. This is an Open Access article distributed under the terms of the Creative Commons Attribution License (http://creativecommons.org/licenses/by/2.0), which permits unrestricted use, distribution, and reproduction in any medium, provided the original work is properly cited. 
where $1<\alpha \leq 2$ is a real number and $f:[0,1] \times \mathbb{R}^{+} \rightarrow \mathbb{R}^{+}$is continuous. They obtained the existence of positive solutions by means of Guo-Krasnosel'skii fixed point theorem and Leggett-Williams fixed point theorem.

In [5], Jiang et al. discussed some positive properties of the Green function for boundary value problem (1.2), and as an application, they utilized the Guo-Krasnosel'skii fixed point theorem to obtain the existence of positive solutions for (1.2).

In [6], El-Shahed and Nieto investigated the existence of nontrivial solutions for a multipoint boundary value problem for fractional differential equations. Under certain growth conditions on the nonlinearity, several sufficient conditions for the existence of a nontrivial solution were obtained by using the Leray-Schauder nonlinear alternative.

In [7], Wang and Liu adopted the same methods in [6] to discuss the existence of solutions for nonlinear fractional differential equations with fractional anti-periodic boundary conditions

$$
\left\{\begin{array}{l}
{ }^{c} D^{\alpha} x(t)=f\left(t, x(t),{ }^{c} D^{q} x(t)\right), \quad t \in[0, T], \\
x(0)=-x(T), \quad{ }^{c} D^{p} x(0)=-{ }^{c} D^{p} x(T) .
\end{array}\right.
$$

In [8-10], Ahmad et al. utilized fixed point theory to consider some fractional differential equations with fractional boundary conditions and obtained some new existence results. In particular, He and his coauthors [10] investigated the existence of solutions for the fractional nonlinear integro-differential equation of mixed type on a semi-infinite interval in a Banach space

$$
\begin{cases}D^{\alpha} u(t)+f(t, u(t), T u(t), S u(t))=\theta, & n-1<\alpha \leq n, n \geq 2, \\ u(0)=u^{\prime}(0)=\cdots=u^{(n-2)}(0)=\theta, & D^{\alpha-1} u(\infty)=u_{\infty} .\end{cases}
$$

Meanwhile, we also note that they developed an explicit iterative sequence for approximating the solution together with an error estimate for the approximation.

In [22, 23], Sun and Zhang discussed a class of singular superlinear and sublinear SturmLiouville problems, respectively. In the two papers, the Sturm-Liouville problems are considered under some conditions concerning the first eigenvalues corresponding to the relevant linear operators, and the nonnegativity is not necessary to be nonnegative. The existence results of nontrivial solutions and positive solutions are given by means of topological degree theory.

Motivated by the works mentioned above, in our paper, we adopt the methods of [22, 23 ] to investigate the fractional problem (1.1). As we know, the eigenvalue and eigenfunction of an integer-order differential equation have been a very perfect theory; however, this work on fractional order differential equation has not appeared in the literature. In order to overcome the difficulty arising from it, we establish some inequalities associated with Green's function; see Lemma 2.3 in Section 2. With the aid of these inequalities, the nonlinear term $f$ can grow superlinearly and sublinearly, and we obtain that problem (1.1) has at least one nontrivial solution by topological degree theory. This means that both our methodology and results in this paper are different from those in $[4-7,11-16]$. 


\section{Preliminaries}

The Riemann-Liouville fractional derivative of order $\alpha>0$ of a continuous function $f$ : $(0,+\infty) \rightarrow(-\infty,+\infty)$ is given by

$$
D_{0+}^{\alpha} f(t)=\frac{1}{\Gamma(n-\alpha)}\left(\frac{\mathrm{d}}{\mathrm{d} t}\right)^{n} \int_{0}^{t} \frac{f(s)}{(t-s)^{\alpha-n+1}} \mathrm{~d} s,
$$

where $n=[\alpha]+1,[\alpha]$ denotes the integer part of number $\alpha$, provided that the right-hand side is pointwise defined on $(0,+\infty)$. For more details on fractional calculus, we refer the reader to the recent books; see [1-3]. Next, we present Green's function of fractional differential equation boundary value problem (1.1).

Lemma 2.1 (See [16, Lemma 2.7]) Let $v \in C[0,1]$ and $\alpha \in(2,3]$. Then $-D_{0_{+}}^{\alpha} u:=v$, together with the boundary conditions $u(0)=u^{\prime}(0)=u^{\prime}(1)=0$, is equivalent to $u(t)=$ $\int_{0}^{1} G(t, s) v(s) \mathrm{d} s$, where

$$
G(t, s):=\frac{1}{\Gamma(\alpha)} \begin{cases}t^{\alpha-1}(1-s)^{\alpha-2}-(t-s)^{\alpha-1}, & 0 \leq s \leq t \leq 1, \\ t^{\alpha-1}(1-s)^{\alpha-2}, & 0 \leq t \leq s \leq 1 .\end{cases}
$$

Lemma 2.2 (See $[16$, Lemma 2.8]) The functions $G(t, s) \in C([0,1] \times[0,1],[0,+\infty))$. Moreover, $G(t, s)$ satisfies the following inequalities:

$$
t^{\alpha-1} s(1-s)^{\alpha-2} \leq \Gamma(\alpha) G(t, s) \leq s(1-s)^{\alpha-2}, \quad \forall t, s \in[0,1] .
$$

Lemma 2.3 Let $\varphi(t)=t(1-t)^{\alpha-2}, \forall t \in[0,1]$, and $\frac{\alpha \Gamma(\alpha-1)}{\Gamma(2 \alpha)}\left(:=\mathcal{K}_{1}\right) \leq \frac{1}{\alpha(\alpha-1) \Gamma(\alpha)}\left(:=\mathcal{K}_{2}\right)$. Then

$$
\mathcal{K}_{1} \varphi(s) \leq \int_{0}^{1} G(t, s) \varphi(t) \mathrm{d} t \leq \mathcal{K}_{2} \varphi(s), \quad \forall s \in[0,1]
$$

Proof By (2.2), we arrive at the inequality (2.3) immediately. The proof is completed.

By simple computation, we have $\max _{t \in[0,1]} \int_{0}^{1} G(t, s) \mathrm{d} s=\int_{0}^{1} \varphi(t) \mathrm{d} t=\frac{1}{\alpha(\alpha-1)}=\mathcal{K}_{2} \Gamma(\alpha)$. Let

$$
E:=C[0,1], \quad\|u\|:=\max _{t \in[0,1]}|u(t)|, \quad P:=\{u \in E: u(t) \geq 0, t \in[0,1]\} .
$$

Then $(E,\|\cdot\|)$ becomes a real Banach space and $P$ is a cone on $E$. Now, note that $u$ solves (1.1) if and only if $u$ is a fixed point of the operator

$$
(A u)(t):=\int_{0}^{1} G(t, s) f(s, u(s)) \mathrm{d} s, \quad u \in E .
$$

Clearly, $f \in C([0,1] \times \mathbb{R}, \mathbb{R})$ implies $A: E \rightarrow E$ is a completely continuous operator. Denote

$$
(L u)(t):=\int_{0}^{1} G(t, s) u(s) \mathrm{d} s, \quad u \in E .
$$

Then $L: E \rightarrow E$ is a completely continuous linear operator, satisfying $L(P) \subset P$. That is, $L$ is a positive, completely continuous, linear operator. Let

$$
P_{0}:=\left\{u \in E: \int_{0}^{1} u(t) \varphi(t) \mathrm{d} t \geq \omega\|u\|, t \in[0,1]\right\},
$$


where $\varphi(t)$ is defined by Lemma 2.3 and $\omega:=\mathcal{K}_{1} \Gamma(\alpha)>0$. By (2.2) and (2.3), we easily have the following result.

Lemma 2.4 $L(P) \subset P_{0}$.

Proof From (2.2), for $u \in P$, we have

$$
(L u)(t)=\int_{0}^{1} G(t, s) u(s) \mathrm{d} s \leq \frac{1}{\Gamma(\alpha)} \int_{0}^{1} \varphi(s) u(s) \mathrm{d} s .
$$

On the other hand, from (2.2) and (2.3), we find

$$
\int_{0}^{1}(L u)(t) \varphi(t) \mathrm{d} t=\int_{0}^{1}\left(\int_{0}^{1} G(t, s) u(s) \mathrm{d} s\right) \varphi(t) \mathrm{d} t \geq \mathcal{K}_{1} \int_{0}^{1} \varphi(s) u(s) \mathrm{d} s \geq \mathcal{K}_{1} \Gamma(\alpha)\|L u\| .
$$

Therefore, $L(P) \subset P_{0}$. This completes the proof.

Lemma 2.5 (See [24]) Let $E$ be a Banach space and let $\Omega \subset E$ be a bounded open set. Suppose that $A: \bar{\Omega} \rightarrow E$ is a completely continuous operator. If there is $u_{0} \neq 0$ such that $u \neq A u+\mu u_{0}, \forall u \in \partial \Omega$ and $\mu \geq 0$, then the topological degree $\operatorname{deg}(I-A, \Omega, 0)=0$.

Lemma 2.6 (See [24]) Let E be a Banach space and let $\Omega \subset E$ be a bounded open set with $0 \in \Omega$. Suppose that $A: \bar{\Omega} \rightarrow E$ is a completely continuous operator. If $A u \neq \mu u, \forall u \in \partial \Omega$ and $\mu \geq 1$, then the topological degree $\operatorname{deg}(I-A, \Omega, 0)=1$.

\section{Main results}

We denote $\lambda_{1}:=\mathcal{K}_{1}^{-1}>0, \lambda_{2}:=\mathcal{K}_{2}^{-1}>0$ and $B_{\rho}:=\{u \in E:\|u\|<\rho\}$ for $\rho>0$.

Theorem 3.1 If there exists $a$ constant $b \geq 0$ such that

$$
f(t, u) \geq-b, \quad \forall u \in \mathbb{R}, \quad \liminf _{u \rightarrow+\infty} \frac{f(t, u)}{u}>\lambda_{1}, \quad \limsup _{u \rightarrow 0}\left|\frac{f(t, u)}{u}\right|<\lambda_{2},
$$

then (1.1) has at least one nontrivial solution.

Proof The first two inequalities of (3.1) imply that there are $\varepsilon>0$ and $b_{1}>0$ such that

$$
f(t, u) \geq\left(\lambda_{1}+\varepsilon\right) u-b_{1}, \quad \forall u \in \mathbb{R}, t \in[0,1] .
$$

Take $R>b \mathcal{K}_{2} \Gamma(\alpha)+\varepsilon^{-1} b\left(\mathcal{K}_{1}^{-3} \mathcal{K}_{2}^{3}-\mathcal{K}_{1}^{-1} \mathcal{K}_{2}\right)+b \mathcal{K}_{1}^{-2} \mathcal{K}_{2}^{3}+\varepsilon^{-1} b_{1} \mathcal{K}_{1}^{-2} \mathcal{K}_{2}^{2}$. In what follows, we shall prove that

$$
u \neq A u+\mu u^{*}, \quad \forall u \in E, \quad\|u\|=R, \quad \mu \geq 0,
$$

where $u^{*} \in P_{0}$. Indeed, if $u_{0} \in E,\left\|u_{0}\right\|=R$, and $\mu_{0} \geq 0$ such that

$$
u_{0}(t)=\left(A u_{0}\right)(t)+\mu_{0} u^{*}(t)=\int_{0}^{1} G(t, s) f\left(s, u_{0}(s)\right) \mathrm{d} s+\mu_{0} u^{*}(t) .
$$


Let $\widetilde{u}(t)=b \int_{0}^{1} G(t, s) \mathrm{d} s$, then we have

$$
u_{0}(t)+\widetilde{u}(t)=\int_{0}^{1} G(t, s)\left(f\left(s, u_{0}(s)\right)+b\right) \mathrm{d} s+\mu_{0} u^{*}(t),
$$

which leads to $u_{0}+\widetilde{u} \in P_{0}$ by Lemma 2.4. Combining this with (3.2), we find

$$
\begin{aligned}
& \int_{0}^{1}\left(A u_{0}\right)(t) \varphi(t) \mathrm{d} t-\int_{0}^{1} u_{0}(t) \varphi(t) \mathrm{d} t \\
&=\int_{0}^{1} \varphi(t) \int_{0}^{1} G(t, s) f\left(s, u_{0}(s)\right) \mathrm{d} s \mathrm{~d} t-\int_{0}^{1} u_{0}(t) \varphi(t) \mathrm{d} t \\
& \geq\left(\lambda_{1}+\varepsilon\right) \int_{0}^{1} \varphi(t) \int_{0}^{1} G(t, s) u_{0}(s) \mathrm{d} s \mathrm{~d} t-b_{1} \int_{0}^{1} \varphi(t) \int_{0}^{1} G(t, s) \mathrm{d} s \mathrm{~d} t-\int_{0}^{1} u_{0}(t) \varphi(t) \mathrm{d} t \\
&=\left(\lambda_{1}+\varepsilon\right) \int_{0}^{1} \varphi(t) \int_{0}^{1} G(t, s)\left(u_{0}(s)+\widetilde{u}(s)\right) \mathrm{d} s \mathrm{~d} t-\left(\lambda_{1}+\varepsilon\right) \int_{0}^{1} \varphi(t) \int_{0}^{1} G(t, s) \widetilde{u}(s) \mathrm{d} s \mathrm{~d} t \\
&-b_{1} \int_{0}^{1} \varphi(t) \int_{0}^{1} G(t, s) \mathrm{d} s \mathrm{~d} t-\int_{0}^{1} u_{0}(t) \varphi(t) \mathrm{d} t \\
& \geq \varepsilon \lambda_{1}^{-1} \int_{0}^{1} \varphi(t)\left(u_{0}(t)+\widetilde{u}(t)\right) \mathrm{d} t+\int_{0}^{1} \widetilde{u}(t) \varphi(t) \mathrm{d} t \\
& \quad-\left(\lambda_{1}+\varepsilon\right) \int_{0}^{1} \varphi(t) \int_{0}^{1} G(t, s) \widetilde{u}(s) \mathrm{d} s \mathrm{~d} t \\
&-b_{1} \int_{0}^{1} \varphi(t) \int_{0}^{1} G(t, s) \mathrm{d} s \mathrm{~d} t \\
& \geq \varepsilon \omega \lambda_{1}^{-1}\left\|u_{0}+\widetilde{u}\right\|+b \mathcal{K}_{1} \mathcal{K}_{2} \Gamma(\alpha)-b\left(\lambda_{1}+\varepsilon\right) \mathcal{K}_{2}^{3} \Gamma(\alpha)-b_{1} \mathcal{K}_{2}^{2} \Gamma(\alpha) \\
& \geq \varepsilon \omega \lambda_{1}^{-1}\left\|u_{0}\right\|-\varepsilon \omega \lambda_{1}^{-1}\|\widetilde{u}\|+b \mathcal{K}_{1} \mathcal{K}_{2} \Gamma(\alpha)-b\left(\lambda_{1}+\varepsilon\right) \mathcal{K}_{2}^{3} \Gamma(\alpha)-b_{1} \mathcal{K}_{2}^{2} \Gamma(\alpha) \\
& \geq \varepsilon \omega \lambda_{1}^{-1} R-\varepsilon \omega \lambda_{1}^{-1} b \mathcal{K}_{2} \Gamma(\alpha)+b \mathcal{K}_{1} \mathcal{K}_{2} \Gamma(\alpha)-b\left(\lambda_{1}+\varepsilon\right) \mathcal{K}_{2}^{3} \Gamma(\alpha)-b_{1} \mathcal{K}_{2}^{2} \Gamma(\alpha)>0 .
\end{aligned}
$$

On the other hand, we have by (3.4)

$$
\int_{0}^{1} u_{0}(t) \varphi(t) \mathrm{d} t-\int_{0}^{1}\left(A u_{0}\right)(t) \varphi(t) \mathrm{d} t=\mu_{0} \int_{0}^{1} u^{*}(t) \varphi(t) \mathrm{d} t \geq 0 .
$$

That is a contradiction. As a result of this, (3.3) holds. Lemma 2.5 gives

$$
\operatorname{deg}\left(I-A, B_{R}, 0\right)=0 .
$$

It follows from the third inequality of (3.1) that there exists $0<r<R$ such that $|f(t, u)| \leq$ $\lambda_{2}|u|, \forall|u| \leq r, t \in[0,1]$. In the following, we prove

$$
A u \neq \mu u, \quad \forall u \in \partial B_{r}, \mu \geq 1 .
$$

In fact, suppose that there exist $u_{1} \in \partial B_{r}, \mu_{1} \geq 1$ such that $A u_{1}=\mu_{1} u_{1}$. We may suppose that $\mu_{1}>1$ (otherwise we are done). Thus

$$
\mu_{1}\left|u_{1}(t)\right| \leq \lambda_{2} \int_{0}^{1} G(t, s)\left|u_{1}(s)\right| \mathrm{d} s .
$$


Multiply by $\varphi(t)$ both sides of the preceding inequality and integrate over $[0,1]$, and use (2.3) to obtain

$$
\mu_{1} \int_{0}^{1}\left|u_{1}(t)\right| \varphi(t) \mathrm{d} t \leq \lambda_{2} \int_{0}^{1} \varphi(t) \int_{0}^{1} G(t, s)\left|u_{1}(s)\right| \mathrm{d} s \mathrm{~d} t \leq \int_{0}^{1}\left|u_{1}(t)\right| \varphi(t) \mathrm{d} t .
$$

This, together with $\int_{0}^{1}\left|u_{1}(t)\right| \varphi(t) \mathrm{d} t>0$, leads to $\mu_{1} \leq 1$, which is a contradiction. So, (3.6) holds. Lemma 2.6 implies

$$
\operatorname{deg}\left(I-A, B_{r}, 0\right)=1 .
$$

By (3.5) and (3.8), we have $\operatorname{deg}\left(I-A, B_{R} \backslash \bar{B}_{r}, 0\right)=\operatorname{deg}\left(I-A, B_{R}, 0\right)-\operatorname{deg}\left(I-A, B_{r}, 0\right)=$ $0-1=-1$. Then $A$ has at least one fixed point on $B_{R} \backslash \bar{B}_{r}$. This means that problem (1.1) has at least one nontrivial solution.

In order to prove Theorem 3.2, we need the following result involving the spectral radius of $L$, denoted by $r(L)$.

Lemma 3.1 $0<r(L) \leq \mathcal{K}_{2}$.

Proof We easily obtain the result by Gelfand's theorem and (2.2). This completes the proof.

Theorem 3.2 If there exists a constant $b \geq 0$ such that

$$
f(t, u) \geq-b, \quad \forall u \in \mathbb{R}, \quad \liminf _{u \rightarrow 0} \frac{f(t, u)}{|u|}>\lambda_{1}, \quad \limsup _{u \rightarrow+\infty} \frac{f(t, u)}{u}<\lambda_{2},
$$

then (1.1) has at least one nontrivial solution.

Proof By the second inequality of (3.9), there exist $\varepsilon>0$ and $r_{1}>0$ such that

$$
f(t, u) \geq\left(\lambda_{1}+\varepsilon\right)|u|, \quad \forall|u| \leq r_{1}, t \in[0,1] .
$$

For every $u \in \bar{B}_{r_{1}}$, we have from (3.10) that

$$
(A u)(t) \geq\left(\lambda_{1}+\varepsilon\right) \int_{0}^{1} G(t, s)|u(s)| \mathrm{d} s, \quad t \in[0,1]
$$

and thus $A\left(\bar{B}_{r_{1}}\right) \subset P$. For all $u \in \partial B_{r_{1}} \cap P$, from (3.10), we know

$$
(A u)(t) \geq\left(\lambda_{1}+\varepsilon\right) \int_{0}^{1} G(t, s) u(s) \mathrm{d} s, \quad t \in[0,1]
$$

We may suppose that $A$ has no fixed point on $\partial B_{r_{1}}$ (otherwise, the proof is completed). Now we show that

$$
u \neq A u+\mu u^{*}, \quad \forall u \in \partial B_{r_{1}} \cap P, \mu \geq 0,
$$


where $u^{*} \in P$. Otherwise, there exist $u_{0} \in \partial B_{r_{1}} \cap P, \mu_{0} \geq 0$ such that $u_{0}=A u_{0}+\mu_{0} u^{*} \geq$ $A u_{0}$. Consequently,

$$
u_{0}(t) \geq\left(A u_{0}\right)(t) \geq\left(\lambda_{1}+\varepsilon\right) \int_{0}^{1} G(t, s) u_{0}(s) \mathrm{d} s
$$

Multiply by $\varphi(t)$ both sides of the preceding inequality and integrate over $[0,1]$, and use (2.3) to obtain

$$
\int_{0}^{1} u_{0}(t) \varphi(t) \mathrm{d} t \geq\left(\lambda_{1}+\varepsilon\right) \int_{0}^{1} \varphi(t) \int_{0}^{1} G(t, s) u_{0}(s) \mathrm{d} s \mathrm{~d} t \geq\left(\lambda_{1}+\varepsilon\right) \lambda_{1}^{-1} \int_{0}^{1} u_{0}(t) \varphi(t) \mathrm{d} t,
$$

which implies $\int_{0}^{1} u_{0}(t) \varphi(t) \mathrm{d} t=0$, and then $u_{0}(t) \equiv 0, \forall t \in[0,1]$. It contradicts $u_{0} \in$ $\partial B_{r_{1}} \cap P$. Hence (3.11) is true. Since $A\left(\bar{B}_{r_{1}}\right) \subset P$, we have, from the permanence property of fixed point index and Lemma 2.5 , that

$$
\operatorname{deg}\left(I-A, B_{r_{1}}, 0\right)=i\left(A, B_{r_{1}} \cap P, P\right)=0,
$$

where $i$ denotes fixed point index on $P$. Recall the definition of $\tilde{u}$. Clearly, $\tilde{u} \in P$ and $A$ : $C[0,1] \rightarrow P-\widetilde{u}$ by (3.9). Define $\widetilde{A} u=A(u-\widetilde{u})+\widetilde{u}, u \in C[0,1]$. We easily find $\widetilde{A}: C[0,1] \rightarrow P$. By the third inequality of (3.9), there exist $r_{2}>r_{1}+\|\tilde{u}\|=r_{1}+b \mathcal{K}_{2} \Gamma(\alpha)$ and $0<\sigma<1$ such that

$$
f(t, u) \leq \sigma \lambda_{2} u, \quad \forall u \geq r_{2}, t \in[0,1]
$$

Let $L_{1} u=\sigma \lambda_{2} L u, \forall u \in C[0,1]$. Then $L_{1}: C[0,1] \rightarrow C[0,1]$ is a bounded linear operator and $L_{1}(P) \subset P$. Let

$$
M=2 \max \left\{\sup _{u \in \bar{B}_{r_{2}}} \int_{0}^{1} G(t, s)|f(s, u(s))| \mathrm{d} s, 2 b \mathcal{K}_{2} \Gamma(\alpha)\right\}<+\infty
$$

and $W:=\{u \in P: u=\mu \widetilde{A} u, 0 \leq \mu \leq 1\}$. In what follows, we will show that $W$ is bounded. For all $u \in W$, let $\widetilde{\psi}(t)=\min \left\{u(t)-\widetilde{u}(t), r_{2}\right\}$ and $e(u)=\left\{t \in[0,1]: u(t)-\widetilde{u}(t)>r_{2}\right\}$. When $u(t)-\widetilde{u}(t)<0, \widetilde{\psi}(t)=u(t)-\widetilde{u}(t) \geq u(t)-r_{2} \geq-r_{2}$, and so $\|\widetilde{\psi}\| \leq r_{2}$. Consequently, for $u \in W$, we have from (3.13)

$$
\begin{aligned}
u(t) & =\mu(\widetilde{A} u)(t) \leq \int_{0}^{1} G(t, s) f(s, u(s)-\widetilde{u}(s)) \mathrm{d} s+\widetilde{u}(t) \\
& =\int_{e(u)} G(t, s) f(s, u(s)-\widetilde{u}(s)) \mathrm{d} s+\int_{[0,1] \backslash e(u)} G(t, s) f(s, u(s)-\widetilde{u}(s)) \mathrm{d} s+\widetilde{u}(t) \\
& \leq \sigma \lambda_{2} \int_{0}^{1} G(t, s) u(s) \mathrm{d} s+\int_{0}^{1} G(t, s) f(s, \widetilde{\psi}(s)) \mathrm{d} s+2 \widetilde{u}(t) \\
& \leq \sigma \lambda_{2} \int_{0}^{1} G(t, s) u(s) \mathrm{d} s+M=\left(L_{1} u\right)(t)+M
\end{aligned}
$$

and then $\left(\left(I-L_{1}\right) u\right)(t) \leq M, t \in[0,1]$. By Lemma 3.1 and $0<\sigma<1, r\left(L_{1}\right)=\sigma \lambda_{2} r(L) \leq$ $\sigma \lambda_{2} \mathcal{K}_{2}<1$. Therefore, the inverse operator $\left(I-L_{1}\right)^{-1}$ exists and $\left(I-L_{1}\right)^{-1}=I+L_{1}+L_{1}^{2}+\cdots+$ 
$L_{1}^{n}+\cdots$. It follows from $L_{1}(P) \subset P$ that $\left(I-L_{1}\right)^{-1}(P) \subset P$. So, we have $u(t) \leq\left(I-L_{1}\right)^{-1} M$, $t \in[0,1]$ and $W$ is bounded.

Select $r_{3}>\max \left\{r_{2}\right.$, sup $\left.W+b \mathcal{K}_{2} \Gamma(\alpha)\right\}$ and thus $\tilde{A}$ has no fixed point on $\partial B_{r_{3}}$. Indeed, if there exists $u_{1} \in \partial B_{r_{3}}$ such that $\widetilde{A} u_{1}=u_{1}$, then $u_{1} \in W$ and $\left\|u_{1}\right\|=r_{3}>\sup W$, which is a contradiction. Then we have from the permanence property and the homotopy invariance property of fixed point index that

$$
\operatorname{deg}\left(I-\widetilde{A}, B_{r_{3}}, 0\right)=i\left(\widetilde{A}, B_{r_{3}} \cap P, P\right)=i\left(0, B_{r_{3}} \cap P, P\right)=1 .
$$

Set the completely continuous homotopy $H(t, u)=A(u-t \tilde{u})+t \tilde{u},(t, u) \in[0,1] \times \bar{B}_{r_{3}}$. If there exists $\left(t_{0}, u_{2}\right) \in[0,1] \times \partial B_{r_{3}}$ such that $H\left(t_{0}, u_{2}\right)=u_{2}$, and then $A\left(u_{2}-t_{0} \widetilde{u}\right)=u_{2}-t_{0} \widetilde{u}$ and $\widetilde{A}\left(u_{2}-t_{0} \tilde{u}+\widetilde{u}\right)=u_{2}-t_{0} \tilde{u}+\widetilde{u}$. Thus $u_{2}-t_{0} \tilde{u}+\tilde{u} \in W$ and $\left\|u_{2}-t_{0} \tilde{u}+\widetilde{u}\right\| \geq\left\|u_{2}\right\|-$ $\left(1-t_{0}\right)\|\tilde{u}\| \geq r_{3}-\|\tilde{u}\|>\sup W$, which is a contradiction. From the homotopy invariance of topological degree and (3.14), we have

$$
\operatorname{deg}\left(I-A, B_{r_{3}}, 0\right)=\operatorname{deg}\left(I-\widetilde{A}, B_{r_{3}}, 0\right)=1
$$

By (3.12) and (3.15), we get $\operatorname{deg}\left(I-A, B_{r_{3}} \backslash \bar{B}_{r_{1}}, 0\right)=\operatorname{deg}\left(I-A, B_{r_{3}}, 0\right)-\operatorname{deg}\left(I-A, B_{r_{1}}, 0\right)=1$, which implies that $A$ has at least one fixed point on $B_{r_{3}} \backslash \bar{B}_{r_{1}}$. This means that the problem (1.1) has at least one nontrivial solution.

Two examples 1 . Let

$$
f(t, u)=a_{1} u+a_{2} u^{2}+\cdots+a_{n} u^{n}, \quad \forall(t, u) \in[0,1] \times \mathbb{R}
$$

where $n$ is a positive even number, $a_{i} \in \mathbb{R}(i=1,2, \ldots, n-1),\left|a_{1}\right|<\lambda_{2}, a_{n}>0$. It is easy to see that $f(t, u)$ is bounded below and usually sign-changing for $u \geq 0$. In addition, $\limsup _{u \rightarrow 0}\left|\frac{f(t, u)}{u}\right|=\left|a_{1}\right|<\lambda_{2}$ and $\liminf _{u \rightarrow+\infty} \frac{f(t, u)}{u}=+\infty$. Thus by Theorem 3.1, we can obtain the existence of a nontrivial solution of (1.1).

2. Let

$$
f(t, u)=\frac{1-u^{2}}{1+u^{2}}, \quad \forall(t, u) \in[0,1] \times \mathbb{R} .
$$

It is easy to see that $f(t, u)$ is bounded below and usually sign-changing for $u \geq 0$. In addition, $\lim \sup _{u \rightarrow+\infty} \frac{f(t, u)}{u}=0<\lambda_{2}$ and $\liminf _{u \rightarrow 0} \frac{f(t, u)}{|u|}=+\infty$. Thus, by Theorem 3.2, we can obtain the existence of a nontrivial solution of (1.1).

Competing interests

The authors declare that they have no competing interests.

Authors' contributions

KZ and JX gave the proof for the main result together. All authors read and approved the final manuscript.

\section{Acknowledgements}

Research is supported by the NNSF-China (10971046), Shandong and Hebei Provincial Natural Science Foundation (ZR2012AQ007, A2012402036), GIIFSDU (yzc12063), IIFSDU (2012TS020). 


\section{References}

1. Podlubny, I: Fractional Differential Equations. Academic Press, San Diego (1999)

2. Kilbas, A, Srivastava, H, Trujillo, J: Theory and Applications of Fractional Differential Equations. Elsevier, Amsterdam (2006)

3. Lakshmikantham, V, Leela, S, Vasundhara Devi, J: Theory of Fractional Dynamic Systems. Cambridge Academic Publishers, Cambridge (2009)

4. Bai, Z, Lü, H: Positive solutions for boundary-value problem of nonlinear fractional differential equation. J. Math. Anal. Appl. 311, 495-505 (2005)

5. Jiang, D, Yuan, C: The positive properties of the Green function for Dirichlet-type boundary value problems of nonlinear fractional differential equations and its application. Nonlinear Anal. 72, 710-719 (2010)

6. El-Shahed, M, Nieto, J: Nontrivial solutions for a nonlinear multi-point boundary value problem of fractional order Comput. Math. Appl. 59, 3438-3443 (2010)

7. Wang, F, Liu, ZH: Anti-periodic fractional boundary value problems for nonlinear differential equations of fractional order. Adv. Differ. Equ. 2012, 116 (2012)

8. Ahmad, B, Nieto, J: Riemann-Liouville fractional differential equations with fractional boundary conditions. Fixed Point Theory 13, 329-336 (2012)

9. Zhang, L, Ahmad, B, Wang, G, Agarwal, RP: Nonlinear fractional integro-differential equations on unbounded domains in a Banach space. J. Comput. Appl. Math. 249, 51-56 (2013)

10. Ahmad, B, Ntouyas, S, Alsaedi, A: A study of nonlinear fractional differential equations of arbitrary order with Riemann-Liouville type multi-strip boundary conditions. Math. Probl. Eng. 2013, Article ID 320415 (2013)

11. Guo, Y: Nontrivial solutions for boundary-value problems of nonlinear fractional differential equations. Bull. Korean Math. Soc. 47, 81-87 (2010)

12. Ferreira, R: Nontrivial solutions for fractional $q$-difference boundary value problems. Electron. J. Qual. Theory Differ. Equ. 2010, $70(2010)$

13. Jia, M, Zhang, $X, G u, X$ : Nontrivial solutions for a higher fractional differential equation with fractional multi-point boundary conditions. Bound. Value Probl. 2012, 70 (2012). doi:10.1186/1687-2770-2012-70

14. Yang, L, Chen, HB: Nonlocal boundary value problem for impulsive differential equations of fractional order. Adv. Differ. Equ. 2011, Article ID 404917 (2011)

15. Sudsutad, W, Tariboon, J: Boundary value problems for fractional differential equations with three-point fractional integral boundary conditions. Adv. Differ. Equ. 2012, 93 (2012)

16. El-Shahed, M: Positive solutions for boundary value problems of nonlinear fractional differential equation. Abstr. Appl. Anal. 2007, Article ID 10368 (2007)

17. Xu, JF, Wei, ZL, Dong, W: Uniqueness of positive solutions for a class of fractional boundary value problems. Appl. Math. Lett. 25, 590-593 (2012)

18. $\mathrm{Xu}, \mathrm{JF}$, Yang, ZL: Multiple positive solutions of a singular fractional boundary value problem. Appl. Math. E-Notes 10 259-267 (2010)

19. Xu, JF, Wei, ZL, Ding, YZ: Positive solutions for a boundary-value problem with Riemann-Liouville's fractional derivative. Lith. Math. J. 52, 462-476 (2012)

20. Wei, ZL, Li, Q, Che, J: Initial value problems for fractional differential equations involving Riemann-Liouville sequential fractional derivative. J. Math. Anal. Appl. 367, 260-272 (2010)

21. Wei, ZL, Dong, W, Che, J: Periodic boundary value problems for fractional differential equations involving a Riemann-Liouville fractional derivative. Nonlinear Anal. 73, 3232-3238 (2010)

22. Sun, J, Zhang, G: Nontrivial solutions of singular superlinear Sturm-Liouville problems. J. Math. Anal. Appl. 313 518-536 (2006)

23. Sun, J, Zhang, G: Nontrivial solutions of singular sublinear Sturm-Liouville problems. J. Math. Anal. Appl. 326, 242-251 (2007)

24. Guo, D, Lakshmikantham, V: Nonlinear Problems in Abstract Cones. Academic Press, Orlando (1988)

doi:10.1186/1687-1847-2013-17

Cite this article as: Zhang and Xu: Nontrivial solutions for a fractional boundary value problem. Advances in Difference Equations 2013 2013:171.

\section{Submit your manuscript to a SpringerOpen ${ }^{0}$ journal and benefit from:}

- Convenient online submission

- Rigorous peer review

- Immediate publication on acceptance

- Open access: articles freely available online

- High visibility within the field

- Retaining the copyright to your article 